\title{
Spektrum der störungsspezifischen verhaltenstherapeutischen Behandlungsansätze bei depressiven Störungen
}

\author{
Aida Buhić-Bergner ${ }^{\mathrm{a}} \quad$ Michael Linden $^{\mathrm{a}, \mathrm{b}}$ \\ a Forschungsgruppe Psychosomatische Rehabilitation, Charité - Universitätsmedizin Berlin, \\ ${ }^{\mathrm{b}}$ Abteilung für psychische und psychosomatische Erkrankungen, \\ Rehabilitationszentrum Seehof der Deutschen Rentenversicherung Bund, Teltow/Berlin, Deutschland
}

\section{Schlüsselwörter}

Störungsspezifische Psychotherapie .

Depressive Störung · Behandlungskonzepte

\section{Zusammenfassung}

In der Diskussion um eine moderne Psychotherapie wird zunehmend eine "störungsspezifische Psychotherapie" gefordert. Diesbezüglich gilt für die Verhaltenstherapie, dass es nicht nur für jede der vielen psychischen Störungen eigene Erklärungsmodelle und Interventionsmöglichkeiten gibt, sondern dass es auch für jede einzelne Störung gleich mehrere Alternativen gibt. Eine störungsspezifische Psychotherapie bedeutet nicht, dass sich aus der Diagnose alleine schon eine störungsspezifische Psychotherapie ableiten ließe, sondern es bedarf in jedem Einzelfall eines patientenspezifischen Störungsmodells und patientenspezifischer Interventionen. Heuristisch leitend müssen dabei die im Rahmen der Verhaltenstherapie über die Jahrzehnte entwickelten evidenzbasierten Störungskonzepte sein. Am Beispiel der depressiven Störung wird eine Übersicht über wichtige unterschiedliche Störungskonzepte und die daraus abgeleiteten Behandlungsmethoden gegeben, d.h. Reinforcer-Modelle, Coping-Modelle, kognitive Modelle, Emotionsmodelle und integrative verhaltenstherapeutische Modelle. Es wird dargestellt, wie diese Theorie- und Behandlungskonzepte auf den einzelnen Fall mittels Verhaltensanalyse zu übertragen sind.

\section{Keywords}

Disorder-specific psychotherapy - Depressive disorder . Therapeutic approaches

\section{Summary}

Spectrum of Disorder-Specific Cognitive-Behavioral Treatment Approaches in Depressive Disorders

In the discussion on modern psychotherapy there is an increasing demand for disorder-specific treatment. In behavior therapy there are many treatments for any disease. The diagnosis does not help in selecting the proper treatment. Instead, a 'patient-specific' treatment has to be developed for every single case, with reference to evidence-based theory and treatment models of cognitive behavior therapy. The following gives an overview of important different concepts of disorder and treatment models for depression, i.e. reinforcer models, coping models, cognitive models, emotion models and integrative behavioral models. It is explained how to apply these different approaches to a patient model based on behavioral analysis.

\section{KARGER \\ Fax +497614520714 \\ Information@Karger.com}

www.karger.com (c) 2013 S. Karger GmbH, Freiburs

$1016-6262 / 13 / 0234-0267 \$ 38.00 / 0$

Accessible online at:

www.karger.com/ver
Prof. Dr. Michael Linden

Rehabilitationszentrum Seehof der Deutschen Rentenversicherung Bund

Lichterfelder Allee 55,14513 Teltow/Berlin, Deutschland

michael.linden@charite.de 


\section{Theorieentwicklung und «Wellen» der Verhaltens- therapie}

Mit den Publikationen zur reziproken Hemmung und systematischen Desensibilisierung [Wolpe, 1958] unter theoretischem Rückgriff auf die Lerntheorie, d.h. auf Modelle des klassischen und operanten Konditionierens, nahm die Verhaltenstherapie (VT) in den 1950er Jahren ihren Anfang. In der folgenden zweiten «Welle» oder «Wende» innerhalb der VT, die in den 1960ern stattfand, wurden sozialpsychologische Paradigmen und Coping-Konzepte in die VT integriert [Goldfried und D’Zurilla, 1969]. Kennzeichnend hierfür ist das Selbstsicherheitstraining bzw. Training der sozialen Kompetenz. In der dritten Welle wurden in den 1970ern mit der Entwicklung von Methoden der Analyse und Modifikation dysfunktionaler Kognitionen wie beispielsweise dem sokratischen Dialog kognitive Theorien aufgegriffen [Mahoney 1974, Ellis 1977, Beck 1976]. Die vierte Welle in den 1980ern war charakterisiert durch die Integration der Emotionstheorien und Entwicklung von Emotionskontrollmethoden wie sie beispielsweise in der Dialektisch Behavioralen Therapie (DBT) [Linehan, 1987] beschrieben werden. In den 1990er Jahren kam als fünfte Welle - in Reaktion unter anderem auf die zunehmende Finanzierung der Psychotherapie durch Krankenkassen - die Entwicklung einer diagnoseorientierten Psychotherapie [Sperry und Carlson, 1996], wonach eine bestimmte Art von Psychotherapie bei einzelnen nach ICD oder DSM definierten Krankheitsbildern positive Wirkungen zu belegen hatte. Die bislang letzte Entwicklungswelle, die nach der Jahrtausendwende eintrat, war durch die neurobiologisch und entwicklungspsychologisch orientierte VT gekennzeichnet [Fuchs, 2004; Etkin et al., 2005; Wilkinson, 2006; Linden 2006, 2008].

Die moderne VT rekurriert gleichzeitig auf alle im Vorangegangenen genannten theoretischen Grundlagen, integriert sie ineinander und entwickelt sie stetig weiter. Jede der VTWellen hat Erklärungs- und Behandlungskonzepte für depressive Störungen ausgearbeitet. Dies hat zur Folge, dass innerhalb der VT nicht nur eine, sondern eine Vielfalt an störungsspezifischen Psychotherapien für Depression zur Verfügung steht. Eine störungsspezifische Psychotherapie kann daher nicht bedeuten, dass sich aus der Diagnose alleine schon eine vorgegebene Art von Psychotherapie ableiten ließe, sondern es bedarf in jedem Einzelfall der Entwicklung eines patientenspezifischen Störungsmodells und patientenspezifischer Interventionen. Heuristisch leitend müssen dabei die im Rahmen der VT über die Jahrzehnte entwickelten evidenzbasierten Störungskonzepte sein. Im Folgenden sollen die wichtigsten kurz überblicksartig dargestellt werden, ohne Anspruch auf Vollständigkeit zu erheben. Für vertiefte Erläuterungen sei auf die Primärliteratur verwiesen. Schließlich soll erläutert werden, wie auf dieser Evidenzbasis ein patientenspezifisches Behandlungskonzept abgeleitet werden kann, mit der Wahl des optimalen verhaltenstherapeutischen Vorgehens im Einzelfall.

\section{Reinforcer- bzw. Verstärker-Modelle}

Verstärkermangel-Modell: Die klassischen lerntheoretischen oder verstärkertheoretischen Erklärungsmodelle für depressive Störungen gehen von der Annahme aus, dass depressives Verhalten durch einen Mangel oder Verlust an positiven Verstärkern (Reinforcer) verursacht und aufrechterhalten wird. Ausgehend von der Skinner'schen funktionalen Verhaltensanalyse entwickelte Ferster [1973] ein operantes Modell, wonach das für Depressive typische Verhalten wie z.B. Antriebsmangel oder depressives Klagen funktional als Vermeidungsverhalten aversiver Stimuli verstanden werden kann. Über die negative Verstärkung und den gleichzeitigen Verlust positiver Verstärkung führt das Vermeidungsverhalten zur Aufrechterhaltung und Intensivierung der depressiven Symptomatik. Auf dieser theoretischen Basis hat Lewinsohn [1974] das Verstärker-Verlust-Modell der Depression ausgearbeitet. Demnach führt depressives Verhalten einerseits zur Löschung positiver Reaktionen des Gegenübers und andererseits wird es kurzzeitig von außen verstärkt [Linden, 1976]. Die wesentliche Konstituente der Lewinsohn'schen Theorie jedoch ist die Annahme, dass Depressive interpersonelle Defizite aufweisen, in dem Sinne, dass ihnen Fertigkeiten und Fähigkeiten fehlen, das Verhalten zu zeigen, das verstärkt werden könnte. In empirischen Untersuchungen wurde gezeigt, dass Depressive eine reduzierte Aktivitätsrate haben, weniger Zufriedenheit aus angenehmen Erlebnissen schöpfen, dass die Stimmung positiv mit angenehmen Erlebnissen und negativ mit unangenehmen korreliert, und dass depressive Personen sich in der verbalen Interaktion langsamer und seltener lobend äußern, d.h. das Verhalten ihrer Interaktionspartner weniger verstärken [Libet und Lewinsohn, 1973; Lewinsohn, 1974; Lewinsohn und Amenson, 1978; Lewinsohn und Talkington, 1979; Grosscup und Lewinsohn, 1980]. Aufgrund dieser Erkenntnisse wurde das Depressionsbewältigungstraining [Lewinsohn et al., 1984] entwickelt, das als primäres Therapieziel angenehme Aktivitäten im Sinne von positiven Verstärkern für nichtdepressives Verhalten sowie die Fähigkeit fördern soll, speziell in sozialen Interaktionen für ein hinreichendes Verstärkerniveau zu sorgen. Therapeutisch werden Listen mit angenehmen Aktivitäten erstellt, und es wird versucht, die Häufigkeit wie auch Stimmungswirksamkeit angenehmer Aktivitäten zu verbessern. In Rollenspielen wird geübt, wie man in Interaktionen positive Verstärkung erzielt [Antonuccio, 1998; Cuijpers et al., 2009]. Aufgrund von Metaanalysen wird für das Depressionsbewältigungstraining eine Effektstärke von 0.28 und in der Prävention eine Risikoreduktion von 38\% geschätzt [Cuijpers, 1998; Cuijpers et al., 2009].

Behaviorale Aktivierungstherapie: Einen verwandten Ansatz verfolgt die Behaviorale Aktivierungstherapie (BA) [Jacobson et al., 1996, 2001; Kanter et al., 2008], derzufolge bei negativer Stimulation durch belastende Lebensumstände oder Alltagsprobleme depressives Verhalten im Sinne eines 
passiven Vermeidungsverhaltens kurzfristig zur Erleichterung führt, d.h. als negative Verstärkung fungiert und damit auf längere Sicht das Verhaltensrepertoire einschränkt und den Zugang zu positiv verstärkenden Verhaltensweisen behindert. Die Therapie konzentriert sich ausschließlich auf den Aufbau neuer Verhaltensweisen. Der Depressive soll lernen, sein Leben so zu gestalten, dass er die eigene Umwelt beeinflussen kann, um den Kontakt mit den Quellen positiver Verstärkung zu erhöhen und den mit negativer Verstärkung zu verringern [Jacobson et al., 2001]. In der BA kommt der Verhaltensanalyse, d.h. der Identifizierung des Kontextes, in dem die Depression auftritt, eine viel bedeutendere Rolle zu als beim traditionellen Aktivitätsaufbau. In der Studie, die letztlich zur Entwicklung der BA führte, verglichen Jacobson et al. [1996] anhand der Werte des Beck-Depressionsinventars (BDI) und der «Hamilton Rating Scale for Depression» (HRSD) die Wirksamkeit der einzelnen Komponenten der kognitiven Therapie nach Beck mit der Wirksamkeit des vollständigen Verfahrens. Die Randomisierung ( $n=149)$ erfolgte folgendermaßen: Die erste Gruppe wurde mit kognitiver Umstrukturierung (AT = automatische Gedanken), die zweite mit Aktivitätsaufbau (BA) und die dritte durch Kombination (CT) der beiden behandelt. In der BA-Gruppe sank der BDI-Wert von 29,3 auf 9,1 und der HRSD-Wert von 17,4 auf 6,4. In der AT-Gruppe sank der BDI-Wert von 29,1 auf 10,6 und der HRSD-Wert von 19,1 auf 6,9, während das vollständige Verfahren zu einer Veränderung auf der BDI-Skala von 29,8 auf 10,1 und auf der HRSD-Skala von 19,1 auf 7,2 führte. Somit ergab die Studie eine vergleichbare Wirkung aller 3 angewendeten Verfahren, die auch im 6-Monats-Follow-up bestand. Dimidjian et al. [2006] verglichen die Wirksamkeit der BA mit kognitiver VT und einer medikamentösen Therapie anhand des BDI-II [Beck et al., 1996]. Bei leichter Depressiven sank der BDIMittelwert durch BA innerhalb von 16 Wochen von 28,7 auf 11, durch CT von 27,3 auf 9,76 und durch die medikamentöse Therapie von 27,79 auf 7,91. Bei schwerer Depressiven sank der BDI-Wert durch BA von 36,7 auf 8,8, durch Medikation von 35,6 auf 7,8 und durch CT von 34,1 auf 17,4. In einer Untersuchung der Rezidivprophylaxe kamen Dobson et al. [2008] zu dem Schluss, dass BA und CT ähnlich wirksam und beide langanhaltender und damit kostengünstiger als die medikamentöse Alternative sind. Auch Metaanalysen ergaben, dass die BA eine wirksame Depressionsbehandlung ist [Mazzucchelli et al., 2009; Cuijpers et al., 2007a, 2008].

Functional analytic psychotherapy (FAP)-enhanced cognitive therapy (FECT): Die FECT baut auf einem radikalen Behaviorismus nach Skinner auf [Kohlenberg und Tsai, 2007; Kohlenberg et al., 1999, 2002]. Die FECT nutzt die Therapiesitzung als soziale Umgebung, in der das klinisch relevante Verhalten (clinically relevant behaviors, CRB) und Kognitionen des Patienten ausgelöst, beobachtet und verändert werden können. Beispielsweise wird ein Patient, der im Alltag seinem Ärger keinen Ausdruck verleihen kann, dieses Verhalten auch in der Therapiesitzung zeigen. Dort wird er vom Therapeuten unmittelbar positiv oder negativ verstärkt. Die Autoren machen eine deutliche und anhaltende Verbesserung der Symptomatik geltend. Allerdings liegt für die FECT nur eine Kohortenstudie vor [Kohlenberg et al., 2002], in der 18 depressive Erwachsene mit kognitiver Therapie behandelt wurden und danach 28 Patienten mit FECT, wobei sich im Vergleich eine größere Reduktion der depressiven Symptomatik und ein besseres Funktionsniveau für die FECT-Gruppe zeigte.

Verstärkerketten-Modell: Eine interessante Weiterentwicklung der Verstärkerkonzepte stellt das Modell des Verlustes von Verstärkereffektivität [Costello, 1972] dar. Es versucht zu erklären, warum objektiv verfügbare Verstärker von Depressiven nicht mehr wahrgenommen oder bei ihnen nicht mehr wirksam werden. Als Erklärung wurde das VerstärkerkettenModell herangezogen, demnach Verstärker in Ketten organisiert sind, sodass beim Ausfall eines Kettengliedes auch alle anderen Verstärker in der Kette ihre verhaltenssteuernde Wirksamkeit verlieren. So bedingt beispielsweise der Verlust des Lebenspartners nicht nur den Wegfall einer Verstärkerquelle, sondern auch den Verlust nachgeordneter Verstärker wie z.B. eines schön gedeckten Tisches oder Theaterbesuchs.

Erlernte Hilflosigkeit: Ebenfalls den Verstärkertheorien zuzuordnen ist die ursprüngliche Theorie der erlernten Hilflosigkeit [Seligman und Maier, 1967; Seligman, 1974]. Hier wird Depression als Folge einer starken und repetitiven aversiven Stimulation bzw. Bestrafung (punishment) gesehen, wobei entscheidend ist, dass der Betroffene keinen Einfluss auf die Beendigung der aversiven Stimulation hat. Es handelt sich also um eine zufällige, «intermittierende», unkontrollierbare Bestrafung. Infolgedessen entsteht eine Negativspirale von Kampf, Flucht, Verzweiflung und letztlich Resignation, die in Apathie, Appetit- und Gewichtsverlust, Initiativmangel und der Unfähigkeit, Neues zu lernen, mündet. Im Rahmen von Experimenten zum hilflosen Verhalten wurde neurobiologisch nach wiederholter aversiver Stimulation ein Noradrenalin-Mangel gemessen, was zur Theorie des motorischen Aktivitätsdefizits und katecholaminergen Defizits führte [Weiss und Glazer, 1975; Weiss et al., 1975]. Demzufolge ist erlernte Hilflosigkeit einem Noradrenalin-Mangel geschuldet und trotz der eindeutig psychologischen Ursache mit Antidepressiva zu behandeln [Glavin, 1985].

\section{Coping-Modelle}

Training sozialer Kompetenz: Bereits in den ReinforcerModellen gilt als ein wichtiger Faktor, inwieweit Patienten über die erforderlichen Fähigkeiten verfügen, sich positive Verstärkung zu sichern. Dies gilt insbesondere für soziale Interaktionen. Unabhängig von der Frage nach Ursache und Wirkung wird bei depressiven Personen eine Einschränkung ihrer sozialen Kompetenzen und Problemlösefähigkeiten be- 
schrieben [Lewinsohn, 1974; Segrin, 2000; Hautzinger, 2013; Bellack et al., 1996; Becker et al., 1987]. Daher bietet sich ein Training der sozialen Kompetenz und der interaktionellen Fähigkeiten an, um den Depressiven einerseits aus seiner sozialen Isolation herauszuführen und um andererseits zu verhindern, dass sich das depressive Interaktions- und Beziehungsmuster depressionsverstärkend auswirkt [Linden, 1976]. Gleiches gilt auch für das Training von Problemlösefertigkeiten. Depressive haben eine Tendenz zu emotionsgesteuerten Problemlösungen, d.h. sie trauen sich weniger zu und gehen Probleme damit nicht hinreichend konsequent an. Das Training sozialer Kompetenzen gehört zum Standardrepertoire der VT. Mit Patienten wird geübt, eigene Wünsche und Rechte zu identifizieren und auszudrücken, Wünsche der Sozialpartner wahrzunehmen, positive wie negative Gefühle auszudrücken, angemessenen Blickkontakt zu halten und eine offene Körperhaltung einzunehmen [Segrin, 2000; Bellack et al., 1996; Becker et al., 1987]. Als wichtigste therapeutische Technik gilt das Rollenspiel [Hautzinger, 2013; Hinsch und Pfingsten, 2002]. In Studien von Bellack et al. [1981] und Hersen et al. [1984] zeigte die Behandlung von unipolarer Depression mit Amitriptylin, mit der kombinierten Behandlung aus sozialem Kompetenztraining und Amitriptylin und einer Kombination von sozialem Kompetenztraining und Placebo die gleiche Wirkung. Am deutlichsten erkennbar war die Reduktion der Symptomatik in der Patientengruppe, die mit dem Training sozialer Kompetenzen und Placebo behandelt wurde. Nach 12-wöchiger Behandlung sank der BDI-Wert von 24,89 auf 7,37.

Problemlösetherapie: Ein über das Training sozialer Kompetenzen hinausgehender Ansatz, der auf die Verbesserung der allgemeinen Problemlösekompetenzen abzielt, wurde von D'Zurilla et al. [1971] vorgestellt. Die Theorie dieses Ansatzes beruht auf dem sozialen Problemlösemodell (PLM) [D'Zurilla und Nezu, 2010; Nezu und Perri, 1989] und dem transaktionalen Problemlösemodell [Lazarus und Launier 1981; Lazarus und Folkman, 1984; Nezu et al., 1989], die Stress als wechselseitigen Prozess zwischen Anforderungen der Umwelt und Bewältigungsvermögen der Person definieren. Eine angemessene, nachhaltige Problemlösekompetenz kann das eigentliche Auftreten, die Art und Anzahl der Probleme beeinflussen, die infolge eines belastenden Lebensereignisses eintreten. Umgekehrt kann eine dysfunktionale Problemlösestrategie zu belastenden Lebensereignissen und Problemzunahme führen. Es wird zwischen 3 Problemlösestilen unterschieden: rational, impulsiv/leichtfertig und vermeidend [D‘Zurilla et al., 2004; D’Zurilla und Nezu, 2010]. Des Weiteren wird zwischen positiver und negativer Problemorientierung unterschieden. Die positive Orientierung und der rationale Lösungsstil gelten als konstruktive Dimensionen, da sie die Wahrscheinlichkeit des positiven Ausgangs erhöhen. Das auf dieser Grundlage entstandene Training zielt darauf $a b$, folgende Problemlösekompetenzen [D'Zurilla et al., 2004] zu vermitteln: Problemdefinition, Entwickeln von Alternativen,
Entscheidungen treffen und Anwendung der Lösung und ihre Überprüfung [Nezu et al., 1989]. Dieses allgemeine Modell der Problemlösekompetenz wurde von Nezu et al. [1989] auf depressive Störungen übertragen. Eine dysfunktionale und ineffiziente Problemlösestrategie steht im Zusammenhang mit der depressiven Entwicklung. Ziele der Therapie sind die Identifikation überfordernder Lebensereignisse und das Training konstruktiver Problemlösekompetenzen einschließlich der Kompensation von negativen Einflüssen der Depression auf Lösungsversuche. Zum Einsatz kommende verhaltenstherapeutische Techniken sind beispielsweise «prompting», «shaping», «behavioral rehearsal», Feedback, Rollenspiele oder In-vivo-Übungen. Bell und D'Zurilla [2009] fanden in einer Metaanalyse von über 21 Studien, dass die Problemlösetherapie (PLT) zu einer Reduktion der depressiven Symptomatik verschiedener Ausprägungsgrade führt. Es wurde festgestellt, dass die PLT gleich wirksam ist wie andere psychosoziale Therapien oder eine medikamentöse Behandlung, wobei ihre Wirksamkeit steigt, je genauer die Therapie technisch umgesetzt wird. Auch eine Metaanalyse von Cuijpers et al. [2007b] bestätigte die Wirksamkeit der PLT. Eine Studie von Mynors-Wallis et al. [1995] ergab zudem, dass die Problemlösetherapie auch als eine wirksame Behandlung der depressiven Störungen in der ärztlichen Grundversorgung eingesetzt werden kann. Die PLT zeigte die größte Response von $60 \%$ auf der HAMD-Skala, im Vergleich zu Patienten, die mit Amitriptylin und Placebo behandelt worden waren (52 und 27\%). In einer weiteren Studie zeigte sich, dass eine Kombination aus PLT und medikamentöser Behandlung keine größere Wirkung zeigt als eine alleinige PLT [MynorsWallis et al., 2000]. Im Vergleich zur Erinnerungstherapie oder einer Warteliste zeigte die PLT in der Behandlung älterer depressiver Patienten die größte Wirksamkeit [Arean et al., 1993].

Interpersonelle Psychotherapie (IPT): Auch die IPT [Klerman et al., 1984; deutsch: Schramm, 1996] ist den Verfahren zum Aufbau von sozialen Kompetenzen und Problemlösekompetenzen zuzurechnen, wobei sie auch auf die psychodynamische Bindungstheorie von Bowlby [1978] und die Interpersonelle Schule von Sullivan [1953] Bezug nimmt. Gemäß dem IPT-Modell ist die Depression eine multifaktoriell bedingte Krankheit, deren Entstehung und Verlauf wesentlich durch belastende Erfahrungen und Erlebnisse im interpersonellen Bereich bestimmt werden. Die IPT-Behandlung zielt trotz der psychodynamischen Theoriebezüge - nicht auf frühe Erfahrungen, sondern auf aktuelle Belastungen, im Sinne von 4 sogenannten Foci. Diese sind Rollenwechsel, interpersonelle Konflikte, pathologische Trauer und interpersonelle Defizite. Das Ziel ist die Verbesserung der zwischenmenschlichen Interaktion mittels Veränderung der Problemlösekompetenzen. Dies beinhaltet die Förderung der Selbstwirksamkeit, der Selbstkontrolle, des Kommunikationsstils oder von Trauerarbeit sowie die Entwicklung der Akzeptanz für neue Rollen. Technische Verfahren sind klassische VT-Interventionen wie 
Kommunikationsanalyse, Rollenspiele, Instruktionen und Hilfen bei Problemlösungen. Eine Metaanalyse von Cuijpers et al. [2008], in der CT, die nondirektive Therapie, BA, die psychodynamische Therapie, Problemlösetherapie, interpersonelle Psychotherapie und ein Training sozialer Kompetenzen miteinander verglichen wurden, ergab für alle eine etwa gleiche Wirksamkeit. In einer Metaanalyse von de Mello et al. [2005], die 13 Studien einschloss, wurde geschlussfolgert, dass die IPT wirksamer ist als Placebo, wirksamer als die CT und in etwa gleich wirksam wie medikamentöse Therapie. Eine randomisierte kontrollierte Studie von Luty et al. [2007] zeigte keinen globalen Unterschied zwischen der IPT und der CT, jedoch wirkte CT in der Gruppe der schwerer depressiven Patienten besser, mit Response-Raten von 57 im Vergleich zu $20 \%$. In der stationären Behandlung depressiver Patienten erwies sich die IPT in Kombination mit einem Antidepressivum im Vergleich zu einer psychiatrischen Standardbehandlung und Medikation als wirksamer [Schramm et al., 2008]. Hinsichtlich der Behandlung von chronischen Formen der Depression erwies sich die IPT alleine weniger wirksam als eine Pharmakotherapie oder die Kombination von Pharmakotherapie und IPT [Markowitz et al., 2005].

\section{Kognitive Modelle}

Bereits in Modellen der klassischen Lerntheorie spielen Kognitionen im Sinne von Erwartungen und erst recht bei sozialpsychologischen Konzepten im Sinne von Bewertungen eine Rolle. Theoretisch ausgearbeitet und in spezifische Therapiekonzepte übertragen wurde die wesentliche Rolle der Kognitionen bei psychischen Störungen erst von Autoren wie Ellis [1977] und Beck [1976], wobei auch hier, so wie beispielsweise bei der IPT, diese Entwicklungen teilweise auf psychodynamischen Erfahrungen aufbauen.

Rational-Emotive Verhaltenstherapie (REVT): Die REVT geht von der Annahme aus, dass die Ursache für die meisten emotionalen Probleme und Verhaltensstörungen in dysfunktionalen kognitiven Prozessen liegt, die sich in Form irrationaler Überzeugungen, Einstellungen und Bewertungen repräsentieren [Ellis 1977, 2008; Dryden, 2005]. Während die rationalen Überzeugungen, die flexibel mit der Wirklichkeit im Verhältnis stehen, logisch und von einer positiven Wirkung auf den Menschen und seine Beziehungen sind und den Kern der psychischen Gesundheit ausmachen, behindern irrationale Überzeugungen die Erreichung der persönlichen Ziele und führen zu psychischen Störungen [Ellis, 2008]. Die REVT baut auf dem A-B-C-Modell auf. Demnach erhält ein auslösendes Ereignis (A: activating event) durch kognitive Bewertungen (B: belief) seine Bedeutung. Daraus lassen sich wiederum emotionale und verhaltensmäßige Konsequenzen (C: consequence) erklären. Ellis benennt auch sogleich die wichtigsten dysfunktionalen Überzeugungen und fasst sie in einer Liste zusammen, so z.B. die Meinung, dass man von jedem geliebt werden müsse, oder dass bestimmte Menschen schlecht seien und bestraft werden müssten. Wenn diese Überzeugungen dogmatisch zu absoluten Forderungen werden (M-Sätze, musturbatorische Ideologie), können sie in einer Depression münden [Di Giuseppe et al., 2002]. Die REVT zielt auf die Identifizierung und Veränderung der irrationalen Überzeugungen, wobei der Betroffene Strategien erlernt, die ihm den zukünftigen Umgang mit Problemen erleichtern sollen. Dabei zielt Ellis nicht nur auf eine symptomatische Verbesserung, sondern auf eine tiefgreifende philosophische Veränderung ab [Ellis, 2008; Dryden und Neenan, 2006]. Entscheidend für die Veränderung und den Therapieerfolg ist, dass der Patient den Zusammenhang zwischen den (irrationalen) Überzeugungen und seinem emotionalen Zustand und Verhalten versteht. Das methodische Kernstück der Therapie ist die Disputation, mit dem Ziel der Hinterfragung von absoluten Forderungen [Ellis, 1980]. Methodisch werden außerdem Imaginationsübungen, Realitätsprüfung, aber auch der Einsatz von Humor oder Metaphern eingesetzt. In ihrem Überblick über Wirksamkeitsbelege der REVT nennen Dryden et al. [2010] 2 Studien. Macaskill und Macaskill [1996] fanden bei Patienten mit einer unipolaren depressiven Episode und hohem Grad kognitiver Dysfunktionalität, dass eine Kombination von REVT mit medikamentöser Therapie besser wirkte als die Medikation allein. Bei chronischen Depressionen zeigte sich die REVT wirksamer als eine medikamentöse Behandlung, wobei die Kombination von beiden noch vielversprechender zu sein schien [Wang et al., 1999; Dryden et al., 2010].

Kognitives Modell nach Beck: Im Gegensatz zu dem vergleichsweise einfachen Modell der REVT, das sich im Wesentlichen auf bewusste Überzeugungen bezieht, erweiterte Beck [1976; Beck et al., 1992; Beck und Alford, 2009] dieses dergestalt, dass es generell einsetzbar wurde. Demnach ist zu unterscheiden zwischen automatischen Gedanken, die vorbewusst sind, aber bewusst gemacht, beobachtet und sogar gezählt werden können, Schemata, d.h. Gruppen von automatischen Gedanken zum selben Thema, die dementsprechend aus der Beobachtung von automatischen Gedanken erschlossen werden können und nicht mit bewussten oder rationalen Überzeugungen verwechselt werden dürfen und schließlich Grundannahmen (basic beliefs), d.h. Wertordnungen mit hoher emotionaler Ladung, die ein Lebensspannen übergreifendes kohärentes Verhalten ermöglichen und auch transgenerational das Leben und Erleben ganzer Gesellschaften steuern. Der psychologische Sinn dieser Kognitionen ist die Reduktion der Komplexität mit dem Ziel einer besseren Alltagsbewältigung und Bedürfnisbefriedigung. Werden Grundannahmen oder Schemata infrage gestellt, werden sie zunächst verteidigt, teilweise mit heftiger emotionaler Beteiligung und gegebenenfalls sogar mithilfe pseudologischer Argumentationen bzw. Denkfehlern wie Magnifizierung, Minimierung, Generalisierung, arbiträre Inferenz, Personalisierung, usw. Im Gegensatz zur REVT, die Kognitionen in rational-irrational und 
richtig-falsch einordnet, unterscheidet die Kognitive Verhaltenstherapie (KVT) je nach Kontext zwischen funktional und dysfunktional. Wem die Familie wichtig ist, wird sich ein Leben lang vor allem um die Familie kümmern und leiden, wenn die Familie zerbricht, aber weniger, wenn die Karriere nicht vorankommt. Wem es im Leben vor allem um die Karriere geht, wird leiden, wenn seine Beförderung in Gefahr ist, während familiäre Probleme möglicherweise als nicht so wichtig erlebt werden. Die KVT greift zum Beginn der Behandlung auf etablierte Verfahren der klassischen VT zurück wie Aktivitätsaufbau oder Selbstbehauptungstraining, um zunächst mit dem Patienten aktuelle Belastungen zu bearbeiten und zu mindern. Der Kern der KVT liegt jedoch in der Umstrukturierung der Kognitionen, die zunächst die Analyse automatischer Gedanken beinhaltet, d.h. averbaler und vorbewusst wertender Wahrnehmungen, die meist mit unmittelbaren emotionalen Reaktionen assoziiert sind. Methodisch bedient man sich der Selbstbeobachtungsaufgaben oder Imaginationsübungen. Im nächsten Schritt wird der emotionale Gehalt typischer automatischer Gedanken herausgearbeitet, z.B. mittels der Spaltentechnik. Es werden im Rahmen von Realitätstestungen alternative Erklärungen für konkrete Situationen gesucht, um eine Reattribution zu erreichen. Letztlich geht es aber darum, die den automatischen Gedanken zugrundeliegenden kognitiven Schemata und Grundannahmen zu modifizieren. Die Methode hierfür ist der Sokratische Dialog, d.h. Hinterfragen und Überprüfen der Zwangsläufigkeit der eigenen Einstellungen und das Zulassen von Alternativen - ein Vorgehen, dass sich wesentlich von Disputation unterscheidet, da Widerspruch eher zur Verhärtung als zur Modifikation von Einstellungen führt. Die Wirksamkeit der KVT bei Depressionen ist in vielen Studien untersucht worden. In der Behandlung akuter depressiver Episoden ist sie wirksamer als Placebo oder supportive Therapien [de JongMeyer et al., 2007]. Eine Metaanalyse von Gloaguen et al. [1998] ergab, dass die KVT bei leichten bis mittelschweren Depressionen wirksamer ist als eine medikamentöse Behandlung oder als eine nichtverhaltenstherapeutische Methode. In der Behandlung von mittelschweren bis schweren Depressionen mit KVT fanden sich Wirkungen, die mit einer medikamentösen Behandlung vergleichbar sind [DeRubeis et al., 2005]. Nach 8 Wochen medikamentöser Behandlung betrug die Response-Rate in dieser Gruppe 50\%, im Vergleich zu $43 \%$ in der Gruppe von Patienten, die mit KVT behandelt worden waren. Nach 12 Wochen betrug die Response-Rate in beiden Gruppen 58\%, die Remissionsrate in der Medikationsgruppe betrug $46 \%$, in der mit kognitiver Therapie $40 \%$. Auch die langfristigen Effekte im Sinne einer Reduktion der Rückfallrate nach Beendigung der Akutbehandlung sind bei kognitiver Therapie besser als bei medikamentöser Therapie. Hollon et al. [2005] verglichen die prophylaktische Wirkung bei 104 Patienten mit mittelschwerer bis schwerer Depression, bei denen entweder KVT oder medikamentöse Therapie wirksam war. Die Behandlung wurde beendet und die Patien- ten in einem Zeitraum von 12 Monaten beobachtet. Die Rückfallrate betrug 30,8\% bei den mit der KVT behandelten Patienten gegenüber 76,2\% in der Medikationsgruppe. In den meisten Vergleichsstudien mit anderen Psychotherapien zeigte sich die KVT sowohl in der Akutbehandlung als auch in der Rückfallprophylaxe den anderen in etwa ebenbürtig [Cuijpers et al., 2008; Dobson et al., 2008; Jacobson et al., 1996].

Schematherapie: Ein weiteres kognitives Modell ist die Schematherapie (ST), die ursprünglich für die Behandlung von Persönlichkeitsstörungen entwickelt, inzwischen aber auch auf Depressionen übertragen wurde [Young, 1990; Young und Matilla, 2002]. Bereits Pretzer und Beck [1996] haben kognitive Psychotherapie und Analyse von kognitiven Schemata auf Persönlichkeitsstörungen angewendet. Ähnlich wie in der REVT, in der schon von Beginn an bekannt ist, unter welchen dysfunktionalen Kognitionen Menschen leiden, werden auch in der Schematherapie die in der Kindheit und späteren Entwicklung ausgebildeten 18 Schemata, die 5 Domänen zugeordnet werden, als allgemeingültig bezeichnet [Young und Matilla, 2002; Young et al., 2005; Sulz, 2007]. Beispiele hierfür sind sichere Bindung zu anderen Menschen, Autonomie, Kompetenz, Identitätsgefühl, Freiheit, berechtigte Bedürfnisse und Emotionen ausdrücken, Spontanität und Spiel sowie eigene Kontrolle [Berbalk und Young, 2009; Young et al., 2005]. Um sich vor Gefahren und Frustrationen zu schützen, in denen Schemata repräsentiert sind, entwickeln Menschen schon früh in der Kindheit maladaptive Bewältigungsstile wie Schemavermeidung, Sich in das Schema fügen oder Schemaüberkompensation. Die aktuell aktiven Schemawirkungen werden im Weiteren sogenannten Schemamodi zugeordnet wie Modi der Kindheit, fehlangepasste Bewältigungsmodi, Elternmodi oder Modi gesunder Erwachsener. Young versteht die ST als Erweiterung der KVT nach Beck [1979] durch Hinzunahme von Erkenntnissen und Techniken aus psychodynamischen, gestalttherapeutischen und bindungstheoretischen Konzepten. Als Interventionsoptionen stehen verhaltenstherapeutische, kognitive, erlebensbasierte Techniken und die therapeutische Beziehung zur Verfügung. Ein Schemadialog zwischen der Schema-Stimme und der gesunden Stimme wird angestoßen, um dem Patienten zu helfen, seinen negativen Emotionen zu begegnen und ihren Ursprung in einem der Schemata zu erkennen, um dadurch die Distanzierung vom eigenen Schema zu erleichtern und die Wahrnehmung und Bewertung der eigenen Kindheit, der eigenen Eltern und aktuellen Umwelt zu verändern. Schließlich werden auch Bewältigungsstile mittels Selbstsicherheitstraining, systematischer Exposition, Rollenspiel, Imagination und Kontingenzmanagement therapeutisch angegangen. Als wirksamste Komponente gilt die therapeutische Beziehung mit empathischer Konfrontation und begrenzter elterlicher Fürsorge, wodurch der Therapeut dem Patienten hilft, seine unerfüllten Kernbedürfnisse zu erkennen und sie zugleich innerhalb der Grenzen der therapeutischen Beziehung zu erfüllen [Young und Matilla 2002; Berbalk und Young, 2009]. Dieses 
Modell wurde auf rezidivierende depressive Episoden und dysthyme Störungen [Young und Matilla, 2002] unter der Annahme übertragen, dass dysfunktionale Schemata im Rahmen depressiver Entwicklungen sich zunehmend verfestigen, dass Schemavermeidung und Schemaüberkompensation das Hinterfragen des eigenen depressiven Selbstbildes und die Generierung von Alternativen verhindern und es zu negativen Interaktionen zwischen Umweltereignissen (z.B. frustrierende Arbeit) und dysfunktionalen Verhaltensmustern kommt. Zur ST in der Behandlung der Depression liegen bislang keine Wirksamkeitsnachweise vor. Young und Matilla [2002] sehen diesen Ansatz dennoch als eine vielversprechende Therapie für chronische und rezidivierende Formen der Depression.

Selbstkontrolltherapie (SKT): Die bisher geschilderten kognitiven Modelle sind attributionstheoretische Modelle, d.h. es geht um inhaltlich bestimmbare dysfunktionale Überzeugungen, Einstellungen und Denkinhalte. Daneben existieren kognitive Modelle, die weniger im Inhalt (was) einer Kognition als vielmehr im Prozess (wie) die Ursache der psychischen Störungen sehen. Demzufolge sind Informationsverarbeitungsprozesse wesentliche Kognitionen. Eines dieser Modelle ist das Selbstkontrollmodell der Depression von Rehm [1977], das sich auf Theorien der Selbstregulation, Selbststeuerung oder des Selbstmanagements bezieht. Nach Kanfer [1970] können Menschen ihr eigenes Verhalten in einem Prozess der Selbstregulation mittels Verstärkung, d.h. mittels Selbstbelohnung bzw. -bestrafung kontrollieren. Dies erfolgt in einem dreistufigen Prozess, der sich aus Selbstbeobachtung, Selbstbewertung und Selbstverstärkung zusammensetzt. Gesunde sind aufgrund der Selbstverstärkung zu einem wesentlichen Teil unabhängig von äußeren Verstärkerquellen, weil sie in der Lage sind, sich selbst zu verstärken. Rehm [1977] versteht Depression als Selbstkontrolldefizit. Depressive geben sich weniger Lob, dafür mehr Selbstbestrafung als Nichtdepressive. Sie haben einen negativen Bewertungsbias sich selbst und ihrem Verhalten gegenüber. Das Problem ist also weniger ein spezifischer Inhalt oder eine benennbare inhaltliche Einstellung, sondern ein allgemeiner Bias in der Informationsverarbeitung und Ereignisbewertung. Auf diesem Modell aufbauend hat Rehm [1977] ein strukturiertes, manualisiertes kognitiv-behaviorales Verfahren für die Behandlung von Depressionen vorgeschlagen, mit dem Hauptziel einer Verbesserung von Selbstkontroll-Fertigkeiten, um dadurch unabhängig von externen Verstärkern zu werden [Rehm und Adams, 2003]. Anhand von Selbstbeobachtungen werden der negative Selbstbewertungsbias herausgearbeitet und positive Selbstverbalisierungen eingeübt, beispielsweise indem der Patient positive Selbstbewertungssätze aufschreibt und sie sich immer wieder vorspricht. Durch Vergleiche von Ist- und Soll-Zustand soll der Patient lernen, sich selbst differenziert zu bewerten. Zum Beleg der Wirksamkeit der SKT bei Depression führten Rehm et al., [1979] eine Vergleichsstudie von SKT und «assertiveness training» durch. Es fand sich sowohl im BDI wie auch auf der «Minnesota Multiphasic Personality
Inventory» (MMPI)-Depressionsskala eine geringere Depressivität bei Patienten in der Selbstkontrolltherapie-Gruppe. Ein Vergleich der Wirksamkeit von SKT alleine und der Kombination aus SKT und Antidepressiva in einer Studie von Roth et al. [1982] zeigte, dass die kombinierte Behandlung zu einer größeren Reduktion des BDI-Werts führte.

Metakognitive Therapie (MCT): Die MCT erklärt die Ursache für die Entstehung und Aufrechterhaltung der Depression mit dysfunktionalen kognitiven Prozessen [Wells und Matthews, 1994; Wells, 2011] und soll sich insbesondere für die Therapie schwerer depressiver Episoden eignen. Eine der zentralen Annahmen ist, dass dysfunktionale kognitive Inhalte, wie sie z.B. von Ellis [1977] und Beck [1976] postuliert werden, ihren Ursprung in Metakognitionen haben, d.h. in Prozessen, die das Denken steuern, kontrollieren und bewerten. Den Kern dieser Selbstregulierungsprozesse bildet das «Cognitive Attentional Syndrome» (CAS), das Metakognitionen wie SichSorgen und Grübeln sowie Bedrohungsmonitoring und maladaptive Bewältigungsstrategien beinhaltet [Wells, 2011; Uhmann und Hoyer, 2011]. Das Verarbeitungsmuster (CAS) wird metakognitiv durch positive und negative Fehlüberzeugungen aufrechterhalten. Betroffene sind zum einen von der Nützlichkeit ihres CAS überzeugt; so glauben sie beispielsweise Folgendes: «Wenn ich mir Sorgen mache, werde ich vorbereitet sein» oder «Wenn ich mögliche Gefahren beachte, bin ich sicher». Zum anderen sind sie von der subjektiv erlebten Unkontrollierbarkeit von Gedanken sowie Gefahren und deren Bedeutung überzeugt, z.B. «Ich habe keine Kontrolle über Sich-Sorgen-Machen und Grübeln» [Wells, 2011]. Die MCT zielt auf eine Bewusstmachung der metakognitiven Prozesse und ihrer Veränderung [Wells, 2009] durch Aufmerksamkeitstraining (ATT) und Üben von losgelöster Achtsamkeit (detached mindfulness) ab, wodurch eine gelassene Kontrolle über das Denken sowie eine gelassene Wahrnehmung der Auslöser des Grübelns erlangt werden sollten. Von Bedeutung sind des Weiteren eine kritische Überprüfung negativer und positiver metakognitiver Überzeugungen in Bezug auf Grübeln, Bedrohungsmonitoring und andere maladaptive Bewältigungsstrategien, das Erarbeiten neuer Pläne der kognitiven Verarbeitung und Rückfallprophylaxe [Wells, 2011]. In einer offenen Studie [Wells et al., 2012] konnte bei depressiven Störungen eine deutliche Besserung der Symptomatik beobachtet werden. Der durchschnittliche BDI-Wert sank von 24 vor der Behandlung auf 7 danach. Den Ergebnissen einer Fallstudie [Wells et al., 2009] zufolge, die 4 Patienten einschloss, führte MCT zur Verbesserung der depressiven Symptomatik, des Grübelns und metakognitiver Überzeugungen. Auch in der Follow-up-Untersuchung blieben die Befunde bestehen.

\section{Emotionsorientierte Therapie}

Mindfulness-Based Cognitive Therapy (MBCT): Die bisher geschilderten Depressionsmodelle sehen die depressive Stim- 
mung als von Verstärkern, Sozialbeziehungen oder kognitiven Prozessen abhängige Variable. Die achtsamkeitsbasierte kognitive Therapie bzw. MBCT stützt sich auf die «differential activation hypothesis» [Teasdale, 1988] und erklärt einen wesentlichen Entstehungs- und Aufrechterhaltungsfaktor von Depression daraus, dass die negative Stimmung dysfunktionale Grundannahmen und negative Gedankenmuster bei ehemals Depressiven schneller aktiviert als bei Gesunden [Lau et al., 2004; Segal et al., 2001, 2008; Teasdale, 1988]. Diese negativen Gedankenmuster (z.B. «Ich bin dumm», «Mir wird nie etwas gelingen») haben einen globalen und stabilen Charakter und führen schon bei geringem Stress zur Exazerbation der negativen Stimmung. Die grüblerische Reaktion ist ein weiterer Risikofaktor. Das als Bewältigungsstrategie eingesetzte Grübeln und Fokussieren nach innen, auf die eigene negative Stimmung, können den Depressionsprozess verstärken und zu einem Rückfall führen [Nolen-Hoeksema, 1987, 2000; Lyubomirski und Tkach, 2003]. Eine hohe dispositionelle Achtsamkeit hingegen kann die Eskalation normalen Nachdenkens ins depressionstypische Grübeln verringern [Raes und Williams, 2010]. Auf Grundlage der genannten Konzepte und mit Bezug auf Elemente des von Kabat-Zinn [1990] entwickelten «Mindfulness-Based Stress Reduction»-Ansatzes entwickelten Segal et al. [2001] die MBCT. Das Therapieziel ist der Erwerb eines distanzierten Verhältnisses zu negativen Gefühlen wie auch Gedanken, wobei Gedanken als Sekundärphänomen interpretiert werden. Mithilfe von Atem-Meditation und Yoga wird Achtsamkeit trainiert, im Sinne einer Fähigkeit, eigene Gedanken und Emotionen mit einer offenen und akzeptierenden Haltung kommen und gehen zu lassen, ohne über diese zu grübeln, sie zu beurteilen oder beeinflussen zu wollen. Durch das Ausrichten der Aufmerksamkeit auf das Hier und Jetzt, durch die Wahrnehmung der körperlichen und seelischen Veränderungen, die von Augenblick zu Augenblick stattfinden, wird verhindert, dass man sich in Gedanken, Erinnerungen sowie in Sorgen um die Zukunft verliert. Eine randomisierte kontrollierte Multizentrumstudie mit Vergleich einer MBCT-Behandlung mit «treatment as usual» (TAU) ergab eine deutliche und statistisch hochsignifikante Reduktion der Rückfallrate bei depressiven Patienten. Die Follow-up-Untersuchung nach einem Jahr ergab, dass 37\% der mit MBCT und 66\% der mit TAU behandelten Patienten einen Rückfall erlitten. Darüber hinaus fanden sich Hinweise, dass MBCT bei Patienten mit weniger als 3 depressiven Episoden nicht wirkt [Teasdale et al., 2000]. Eine Studie von Lau et al. [2004] ergab, dass die MBCT dort am wirksamsten war, wo kein auslösendes Ereignis eine Rolle spielte. In einer Studie von Bondolfi et al. [2010] fand sich kein Unterschied in der Rückfallquote beider Gruppen (MBCT $33 \%$, TAU 36\%). Des Weiteren erwies sich die MBCT als effektiver in der Rückfallprävention als eine Erhaltungstherapie mit Antidepressiva (47 gegenüber 60\%) [Michalak et al., 2008; Kuyken et al., 2008, 2010].

Akzeptanz- und Commitmentherapie (ACT): Die ACT wird von deren Vertretern als Weiterentwicklung des
Skinner'schen Behaviorismus gesehen und bezieht sich in ihrer Theorie partiell auf die kognitive Therapie und insbesondere die Verhaltensanalyse mit dem speziellen Konzept eines sogenannten funktionalen Kontextualismus [Hayes et al., 1999]. Mangelnde Distanz zu kognitivem Erleben, Bewertungsurteile über eigene Erfahrungen, Vermeidung von Erinnerungen an eigene Erfahrungen, Ursachensuche für Geschehenes und der Kampf gegen das, was nicht sein darf oder sollte wird als pathogen angesehen. Dementsprechend wird in der ACT versucht, Negativerleben nicht zu ändern, sondern dem Patienten beizubringen, negative Gefühle und Gedanken so zu nehmen wie sie sind und zu akzeptieren, was nicht zu ändern ist. Deshalb kann sie den emotionsorientierten Verfahren zugeordnet werden. Gleichzeitig wird aber auch vermittelt, Probleme engagiert anzugehen (commitment), die unter den gegebenen Umständen änderbar sind. Die ACT hat daher viele Ähnlichkeiten mit Symptomtoleranzübungen im Rahmen von Expositionstherapien, dem metakognitiven Ansatz, der MBCT oder der Weisheitstherapie [Baumann und Linden, 2008], die unter anderem ebenfalls vermitteln, was nicht zu ändern ist zu akzeptieren und zu ändern, was änderbar ist. Es wird mit einem breiten Spektrum an Interventionen gearbeitet, die zum Ziel haben, die Leidenstoleranz zu erhöhen; von Entspannungs- und Meditationsverfahren, über «Reframing»-Strategien mit Anspruchsrelativierung, Änderung kognitiver Schemata und Wertüberzeugungen, bis hin zur Initiierung von Aktivitäten zur Erhöhung des eigenen Lebensinhalts, kompensatorisch zu den unveränderbaren Negativaspekten.

Die ACT ist kein störungsspezifischer Behandlungsansatz im Sinne einer Depressionstherapie, sondern richtet sich nahezu an das gesamte Spektrum psychischer Störungen - von Schizophrenie über Angststörungen, Schmerz, Abhängigkeitserkrankungen und Essstörungen bis hin zur Epilepsie [Ruiz, 2010]. Es gibt auch erste Arbeiten zum Thema Depression [Forman et al., 2007; Zettle et al., 2011], in denen mittlere Effekte berichtet wurden.

Well-Being Therapy (WBT): Ein weiterer emotionsorientierter Ansatz der VT bei Depression ist die Wohlbefindenstherapie (WBT) [Fava et al., 1998; Fava und Ruini, 2003; Fava et al., 2009]. Zur Behandlung depressiver Störungen wird an der kompensatorischen Förderung des Wohlbefindens gearbeitet. Dabei gilt, dass gute und schlechte Stimmung nicht die entgegengesetzten Pole einer Dimension sind, sondern dass zur Förderung von guter Stimmung eigene Interventionen benötigt werden, die sich von Maßnahmen zur Minderung schlechter Stimmung unterscheiden. Nach Ryff [1989] sind Bedingungen des Wohlbefindens Autonomie, persönliche Entwicklung, Kompetenz im Umgang mit der Umwelt, Lebenssinn, positive Beziehungen und Selbstakzeptanz, weshalb diese Therapie auch einen großen kognitiven Anteil enthält. Die darauf aufbauende WBT [Fava et al., 1998; Fava und Ruini, 2003; Ruini und Fava, 2004; Fava et al., 2009] beginnt nicht mit der Selbstbeobachtung schlechter Phasen, sondern 
mit derer der guten Stimmung. Es wird herausgearbeitet, welche Bereiche des psychologischen Wohlbefindens beeinträchtigt sind und welche Kontingente, seien es Umweltfaktoren oder Kognitionen, positives Befinden fördern. Je nach Einzelfall kann mit Listen angenehmer Aktivitäten, Selbstsicherheitstraining oder der Vermittlung von Problemlösestrategien gearbeitet werden, um Phasen des Wohlbefindens zu stärken. In einer Vergleichsstudie der WBT mit klassischer KVT bei partiell remittierten affektiven Störungen zeigte sich die WBT hinsichtlich Besserung der Befindlichkeit und der RezidivRaten (25 vs. $80 \%$ ) der KVT überlegen. Sie wird von den Autoren vor allem zur Behandlung chronischer depressiver Störungen empfohlen [Fava et al., 1998].

\section{Integrative Modelle}

Wie bei der Beschreibung der bisherigen Therapieansätze bereits erkennbar wurde, fließen die eingangs genannten verschiedenen theoretischen Grundkonzepte der VT (Lerntheorie, Sozialpsychologie, kognitive Theorie, Emotionstheorie) in die verschiedenen Therapiekonzepte in unterschiedlicher Form ein. Jede neue Welle der VT hat die bisherigen Theorie- und Therapieansätze nicht verworfen, sondern sie mit eingeschlossen und gegebenenfalls weiterentwickelt, wodurch sie zunehmend integrativ werden; dies allerdings in einem verhaltenstherapeutischen heuristischen und technologischen Rahmen. Theorien unterschiedlicher Therapieschulen werden nicht gemischt und theoretische Konstrukte nicht aus ihrem Theoriezusammenhang herausgenommen.

Cognitive Behavioral Analysis System of Psychotherapy $(C B A S P)$ : Ein Beispiel für derartig verhaltenstherapeutischintegrative Verfahren ist das CBASP, das speziell für die Behandlung von chronischen Depressionen entwickelt wurde [McCullough, 2006]. Das CBASP basiert laut McCullough explizit auf der Theorie des operanten Lernens nach Skinner [1930], der Theorie der erlernten Hilflosigkeit von Seligman [1974], Banduras Theorie des sozialen Lernens [1977], Kieslers Modell zur interpersonellen Theorie [1996] und den entwicklungspsychologischen Theorien der kognitiv-emotionalen Entwicklung von Piaget [1995]. Es wird angenommen, dass Depressive entwicklungspsychologisch in einer präoperatorischen Entwicklungsphase verharren [Piaget, 1995; McCullough, 2006]. Diese zum Stillstand gekommenen Entwicklungsprozesse resultieren in globalen und prälogischen kognitiven Prozessen und der Unfähigkeit, sich empathisch zu verhalten. Die Unfähigkeit, eigene Emotionen zu regulieren, beeinträchtigt die Funktionsfähigkeit des Patienten in der Familie sowie im Beruf und kommt insbesondere im interpersonellen Bereich negativ zum Tragen. Mangelnde Bewältigungsstrategien in Belastungssituationen führen zum Gefühl der Hoffnungslosigkeit und Hilflosigkeit [McCullough, 2006]. Mittels behavioraler, kognitiver und interpersoneller Techniken zielt die Therapie auf den Erwerb von reiferen, differen- zierteren Denkstrukturen und Verhaltensweisen ab wie z.B. Entwicklung bzw. Verbesserung der Empathiefähigkeit, dysfunktionaler Beziehungsmuster sowie Erwerb der Fähigkeit, die Konsequenzen des eigenen Verhaltens in der Umwelt wahrzunehmen und das Verhalten dementsprechend funktional zu ändern [McCullough, 2006]. Im Kern handelt es sich beim CBASP daher um eine klassisch verhaltensanalytisch geleitete Therapie nach einem erweiterten S-O-R-C-Schema. Die Haupttechnik des CBASP ist dementsprechend die Situationsanalyse, die aus einer Erhebungs- und einer Lösungsphase besteht. Verhaltensdefizite werden mit klassischem Verhaltenstraining, Rollenspielen, gezielter Verstärkung, interpersonellen Diskriminationsübungen und Übungen von Beziehungsmustern in direkter Interaktion zwischen Patient und Therapeut (disciplined personal involvement) verbessert. Eine randomisierte Multizentrumstudie [Keller et al., 2000], die eine medikamentöse Therapie mit Nefazodon, 16-20 Sitzungen CBASP und eine Kombination von beiden verglich, ergab, dass CBASP als Monotherapie gleich wirksam wie eine Pharmakotherapie und weniger wirksam als die Kombination beider Verfahren ist. Nemeroff et al. [2003] argumentieren, dass Patienten mit chronischer Depression und einem Kindheitstrauma (early onset) vom CBASP mehr profitieren als von der Behandlung mit Nefazodon und auch mehr als Patienten ohne traumatische Erfahrungen. In einer Vergleichsstudie [Schramm et al., 2010] fand sich unter CBASP eine höhere Remissionsrate als unter IPT (57 vs. 20\%). Klein et al. [2004] untersuchten den Einsatz des CBASP als Erhaltungstherapie und fanden niedrigere Rückfallraten als in der Patientengruppe, die nur übliche Untersuchungstermine erhielt. Kocsis et al. [2009] untersuchten die Wirksamkeit von CBASP, eines Medikationswechsels und einer kurzen unterstützenden Therapie als Augmentationsbehandlung bei Nonrespondern auf Medikation; sie fanden keinen Unterschied.

\section{Schlussfolgerung}

Aus der vorliegenden Darstellung der verschiedenen verhaltenstherapeutischen Ansätze für depressive Störungen wird deutlich, dass es «die eine» störungsspezifische VT nicht gibt. Fachpolitische Forderungen nach der einen evidenzbasierten störungsspezifischen Psychotherapie greifen zu kurz und ignorieren die Vielfalt der zur Verfügung stehenden Konzepte, nicht nur in unterschiedlichen Therapieverfahren, sondern auch innerhalb der VT.

Die vorliegende Übersicht zeigt, dass es eine kontinuierliche Entwicklung der theoretischen Grundlagen und therapeutischen Interventionen über die Jahre und Wellen hin gibt. Manches ist bei genauerer Betrachtung auch nur bedingt eine Neuentwicklung; vielmehr wird alter Wein in neue Schläuche gegossen, häufig verbunden mit Marketing- und Sales-Intentionen, die es Psychotherapie-Gurus ermöglichen, eine Gemeinde der Gläubigen um sich zu versammeln und über Meis- 
terklassen und Zertifikate Geld zu verdienen. Die geschilderten neuen Therapievarianten haben immer auch die bereits vorliegenden Kenntnisse mitberücksichtigt und integriert. So werden in neuen Therapieansätzen immer auch Aktivitätsaufbau, Problemlösestrategien oder kognitive Modifikation als Teil des Behandlungsregimes genannt. Es handelt sich insofern nicht um distinkte neue Therapien, sondern um Ausdifferenzierungen und Weiterentwicklungen des einen Verfahrens VT. Entsprechend müssen Therapeuten durchgängig über das gleiche methodische Grundrepertoire der VT verfügen - vom Aktivitätsaufbau, über Problemlösestrategien bis hin zur Analyse von Kognitionen oder Verfahren der Emotionswahrnehmung und -beeinflussung.

Die Auswahl des optimalen Vorgehens im Einzelfall erfolgt über die Verhaltensanalyse. Dabei ist zunächst einmal anzumerken, dass sich die Psychotherapie, ebenso wie die Pharmakotherapie, nicht an Diagnosen gemäß der ICD orientiert, sondern an Funktions-, Fähigkeits- und Kontextstörungen gemäß der «International Classification of Functioning, Disability and Health» (ICF) [WHO, 2001]. In der Pharmakotherapie gilt seit ihren Anfängen das Konzept der sogenannten Zielsyndrome [Freyhan, 1960]. Demzufolge stehen für Unruhezustände Tranquilizer, für produktiv psychotische Syndrome Neuroleptika und für Stimmungsprobleme Antidepressiva zur Verfügung, und dies ganz unabhängig davon, in welchem diagnostischen Kontext die Symptome jeweils auftreten. Auch wenn ICD- und DSM-Kategorien in Therapiestudien aufgrund von Anforderungen der amerikanischen «Food and Drug Administration» (FDA) unverzichtbar geworden sind, ist die Pharmakotherapie im Einzelfall weiterhin ausschließlich an Zielsyndromen orientiert.

Auch die Psychotherapie, sei es Tiefenpsychologie, VT oder andere Verfahren, hat sich in diesem Sinne seit jeher auf Leitsyndrome oder Leitprobleme konzentriert. Wer Schwierigkeiten in der sozialen Interaktion hat, erhält ein Training der sozialen Kompetenz, unabhängig davon, ob eine Schizophrenie, Angststörung, Depression oder Persönlichkeitsstörung vorliegt. Da psychische Erkrankungen jedoch nicht monodimensional auftreten oder zu verstehen sind, müssen jeweils individuelle Störungsprofile herausgearbeitet und darauf abgestellt individuelle Behandlungspläne entwickelt werden. Gleichsetzungen und Empfehlungen wie z.B. DBT für Borderline, Schematherapie für Persönlichkeitsstörung oder CBASP für Depression sind fachliche Verkürzungen und erlauben keine verhaltenstherapeutisch adäquate Behandlung.

Das Hauptproblem einiger der zuvor beschriebenen Therapieansätze besteht in Phänomenen, die zur Kernsymptomatik des depressiven Syndroms gehören (z.B. Grübeln, Empathiemangel, Interaktionsprobleme, Passivität, Rückzug), und die Behandlung zielt darauf ab, diese zu bessern. Diese Therapieansätze sind im eigentlichen Sinne also keine Kausalmodelle, auch wenn z.B. im metakognitiven Modell Grübeln als eine der Ursachen und nicht nur als Symptom diskutiert wird. Letztlich handelt es sich um symptomorientierte Konzepte.
Ein anderer Teil der Störungsmodelle geht über die Symptomatik hinaus und fokussiert auf Vulnerabilitäts- oder Resilienzfaktoren wie z.B. Verstärker, Problembewältigungskompetenzen oder Kognitionen. Wie empirische Untersuchungen zeigen, kann auch über diesen Weg eine Besserung depressiver Störungen erreicht werden.

Für die Praxis der VT ergeben sich aus der Vielfalt der Störungsmodelle mehrere Konsequenzen. Zum einen benötigen Verhaltenstherapeuten ein breites Theoriewissen. So ist es wichtig z.B. den Unterschied zwischen den Kognitionsmodellen von Ellis versus Young, versus Beck, versus Rehm und versus Wells zu verstehen. Wer nur ein Modell kennt oder nicht weiß, wie sich diese unterschiedlichen psychischen Phänomene beim Patienten darstellen und objektivieren lassen, ist in seinen therapeutischen Fähigkeiten eingeschränkt und nicht in der Lage, eine individualisierte VT nach dem aktuellen Kenntnisstand durchzuführen. Bei der Supervision von Psychotherapeuten in Ausbildung, die gerade ein Seminar zu einem bestimmten Theorieansatz gehört haben, ist regelhaft zu sehen, dass die Therapeuten mangels alternativer theoretischer Konzepte jeden Patienten dann nach dem gleichen Schema interpretieren.

Des Weiteren gilt, dass nicht jeder depressive Patient mit jedem Theorieansatz adäquat zu beschreiben ist: Nicht jeder Depressive hat mit interpersonellem Verlust- oder Beziehungsproblemen zu kämpfen, nicht jeder hat Lebensbelastungen, nicht jeder hat dysfunktionale kognitive Schemata, usw. Die beschriebenen Konzepte treffen somit nicht für jeden $\mathrm{Pa}$ tienten zu. Sie können aber wie Subdiagnosen depressiver Störungen behandelt werden, beispielsweise im Sinne einer Ferster-, Costello-, Beck- oder Wells-Depression, usw. Die Aufgabe des Therapeuten ist, im Einzelfall zu klären, welches der verschiedenen theoretischen Konzepte und Behandlungsmodelle den «besten Fit» hat. Da in einer Psychotherapie mit begrenzter Stundenzahl nicht alles gleichzeitig angegangen werden kann, ist therapeutisch zu klären, welches Modell am ehesten die psychologische Situation des Patienten abbildet und daher auch den besten Erfolg erwarten lässt.

Schließlich zeigt die Übersicht, dass VT weiterhin das ist, was sie schon immer war: die Entwicklung eines individuellen Störungsmodells mithilfe der Verhaltensanalyse unter Rückgriff auf theoretisches Grundlagenwissen. Verkürzt könnte gesagt werden, dass VT die Anwendung der Verhaltensanalyse ist, d.h. die Beschreibung von Verhaltensabläufen und Verhaltenskontingenten im Bereich von ca. $400 \mathrm{msec}$. Wenn es Soll-Sätze gibt, die vor Jahren gelernt wurden, dann müssen sie in diesen 400 msec z.B. als automatische Gedanken wirksam werden. Wenn ein Trauma, das Jahre zurückliegt, als verhaltensrelevant angesehen werden soll, dann muss es in diesen $400 \mathrm{msec}$ in Form von Emotionserinnerungen aufscheinen. Historisierende biographische Erklärungen oder die sogenannte Makroanalyse können im verhaltenstherapeutischen Sinne keine Verhaltenserklärungen liefern. Mittels der Mikroanalyse lässt sich im konkreten Einzelfall klären, ob Ver- 
stärkerpläne für inadäquates Verhalten vorliegen, frühere Erfahrungen über Erinnerungen oder dysfunktionale Kognitionen im Hier und Jetzt wirksam werden und Copingdefizite oder idiosynkratische Emotionslagen bestehen. Je nach Ergebnis der Verhaltensanalyse kann ein individuelles Störungsmodell erstellt, auf seine Vorhersagekraft durch Verhaltensexperimente und -proben in unterschiedlichen Situationen überprüft und schließlich in sehr individuelle Behandlungspläne übersetzt werden. VT macht, wer verhaltensanalytisch arbeitet. Abhängig von der Konstellation des Einzelfalls kann auf die beschriebenen Therapiekonzepte rekurriert werden. Die Theorie- und Behandlungskonzepte dienen also als Hintergrundmatrizen für den Einzelfall und nicht als schematische Vorschrift. Die genannten Schlussfolgerungen ließen sich vermutlich auch für Angsterkrankungen, Zwangsstörungen, Persönlichkeitsstörungen, usw. ziehen.

\section{Dank}

Die Autoren bedanken sich bei Prof. Dr. M. Hautzinger (Tübingen) für die Durchsicht und Kommentierung des Manuskripts.

\section{Disclosure Statement}

Die Autoren erklären hiermit, dass keine Interessenskonflikte in Bezug auf das Manuskript bestehen.

\section{Literatur}

Antonuccio DO: The coping with depression course: a behavioral treatment for depression. The Clinical Psychologist 1998;51:3-5.

- Arean PA, Perri MG, Nezu AM, Schein RL, Christopher F, Joseph TX: Comparative effectiveness of social problem-solving therapy and reminiscence therapy as treatment for depression in older adults. J Consult Clin Psychol 1993;6:1003-1010.

Bandura A: Social Learning Theory. New York, General Learning Press, 1977.

Baumann K, Linden M: Weisheitskompetenzen und Weisheitstherapie. Lengerich, Pabst, 2008.

Beck AT: Cognitive Therapy and the Emotional Disorders. Boston, International Universities Press, 1976.

Beck AT: Cognitive Therapy of Depression. New York, Guilford Press, 1979

Beck AT, Alford BA: Depression. Causes and Treatment. Philadelphia, University of Pennsylvania Press, 2009.

Beck AT, Steer RA, Ball R, Ranieri WF: Comparison of Beck Depression Inventories -IA and -II in psychiatric outpatients. J Pers Assess 1996;67:588-597.

Beck AT, Rush AJ, Shaw BF, Emery G: Kognitive Therapie der Depression. Weinheim, Psychologie Verlags Union, 1992.

Becker RE, Heimberg RG, Bellack AS: Social Skills Training Treatment for Depression. New York, Pergamon, 1987.

Bell AC, D'Zurilla TJ: Problem-solving therapy for depression: a meta-analysis. Clin Psychol Rev 2009; 29:348-353.

Bellack AS, Hersen M, Himmelhoch JM: Social skills training compared with pharmacotherapy and psychotherapy in the treatment of unipolar depression. Am J Psychiatry 1981;138:1562-1567.

Bellack AS, Hersen M, Himmelhoch JM: Social skills training for depression. A treatment manual; in Van Hasselt VB, Hersen M (eds): Sourcebook of Psychological Treatment Manuals for Adult Disorders. New York, Plenum Press, 1996, pp 179-200.

Berbalk HH, Young JE: Schematherapie; in Margraf J, Schneider S (ed): Lehrbuch der Verhaltenstherapie. Heidelberg, Springer, 2009, pp 645-667.

Bondolfi G, Jermann F, der Linden MV, Gex-Fabry M, Bizzini L, Rouget BW, Myers-Arrazola L, Gonzalez C, Segal Z, Aubry JM, Bertschy G Depression relapse prophylaxis with mindfulnessbased cognitive therapy: replication and extension in the Swiss health care system. J Affect Disord 2010;122:224-231.
Bowlby J: Attachment theory and its therapeutic implications. Adolesc Psychiatry 1978;6:5-33.

Costello CG: Depression: the loss of reinforcers or loss of reinforcer effectiveness? Behav Ther 1972;3: 240-247.

Cuijpers P: A psychoeducational approach to the treatment of depression: a meta-analysis of Lewinsohn's 'coping with depression' course. Behav Ther 1998;29:521-533.

Cuijpers P, Munoz RF, Clarke GN, Lewinsohn PM: Psychoeducational treatment and prevention of depression. Clin Psychol Rev 2009;29:449-458.

Cuijpers P, van Straten A, Andersson G, van Oppen P: Psychotherapy for depression in adults: a metaanalysis of comparative outcome studies. J Consult Clin Psychol 2008;76:909-922.

Cuijpers P, van Straten, Warmerdam L: Behavioral activation treatments of depression: a meta-analysis. Clin Psychol Rev 2007a;27:318-326.

Cuijpers P, van Straten A, Warmerdam L: Problemsolving therapies for depression: a meta-analysis. Eur Psychiat 2007b;22:9-15.

de Jong-Meyer R, Hautzinger M, Kühner C, Schramm E: Evidenzbasierte Leitlinien zur Psychotherapie Affektiver Störungen. Göttingen, Hogrefe, 2007.

de Mello MF, de Jesus Mari J, Bacaltchuk J, Verdeli $\mathrm{H}$, Neugebauer R: A systematic review of research findings on the efficacy of interpersonal therapy for depressive disorders. Eur Arch Psychiatry Clin Neurosci 2005;255:75-82.

DeRubeis RJ, Hollon SD, Amsterdam JD, Shelton RC, Young PR, Salomon RM, O'Reardon JP, Lovett ML, Gladis MM, Brown LL, Gallop R: Cognitive therapy vs. medications in the treatment of moderate to severe depression. Arch Gen Psychiatry 2005;62:409-416.

Di Giuseppe R, Doyle KA, Rose RD: Rational emotive behavior therapy for depression: achieving unconditional self-acceptance; in Reinecke MA, Davison MR: Comparative Treatments of Depression. New York, Springer, 2002.

Dimidjian S, Hollon SD, Dobson KS, Schmaling KB, Kohlenberg RJ, Addis ME, Gallop R, McGlinchey JB, Markley DK, Gollan JK, Atkins DC, Dunner DL, Jacobson NS: Randomized trial of behavioral activation, cognitive therapy, and antidepressant medication in the acute treatment of adults with major depression. J Consult Clin Psychol 2006;74: 658-670.
Dobson KS, Hollon SD, Dimidjian S, Schmaling KB, Kohlenberg RJ, Galopp RJ, Rizvi SL, Gollan JK, Dunner DL, Jacobson NS: Randomized trial of Behavioral Activation, Cognitive Therapy, and antidepressant medication in the prevention of relapse and recurrence in major depression. J Consult Clin Psychol 2008;76:468-477.

Dryden W: Rational emotive behavior therapy; in Freeman A (ed): Encyclopedia of Cognitive Behavior Therapy. New York, Springer, 2005, pp 321- 324.

Dryden W, Neenan M: Rational Emotive Behaviour Therapy. 100 Key Points and Techniques. New York, Routledge, 2006.

Dryden W, David D, Ellis A: Rational emotive behavior therapy; in Dobson KS (ed): Handbook of Cognitive-Behavioral Therapies. New York, Guilford Press, 2010, pp 226-276.

D'Zurilla TJ, Nezu AM: Problem-solving therapy; in Dobson KS (ed): Handbook of Cognitive Behavioral Therapies. New York, Guilford Press, 2010, pp 197-225.

D'Zurilla TJ, Nezu AM, Maydeu-Olivares A: Social problem solving: theory and assessment; in Chang EC, D'Zurilla TJ, Sann LJ: Social Problem Solving: Theory, Research, and Training. Washington D.C., APA, 2004.

D'Zurilla TJ, Thomas J, Goldfried MR, Marvin R: Problem solving and behavior modification. J Abnorm Psychol 1971;78:107-126.

Ellis A: Die rational-emotive Therapie. München, Pfeiffer, 1977.

Ellis A: Rational-emotive therapy and cognitive behavioral therapy: similarities and differences. Cognitive Ther Res 1980;4:325-340.

Ellis A: Grundlagen und Methoden der rational-emotiven Verhaltenstherapie. Stuttgart, Klett-Cotta, 2008.

Etkin A, Pittenger C, Polan HJ, Kandel ER: Toward a neurobiology of psychotherapy: basic science and clinical applications. J Neuropsychiatry Clin Neurosci 2005;17:145-158.

Fava GA, Rafanelli C, Cazzaro M, Conti S, Grandi S: Well-being therapy. A novel psychotherapeutic approach for residual symptoms of affective disorders. Psychol Med 1998;28:475-480.

Fava GA, Ruini C: Development and characteristics of a well-being enhancing psychotherapeutic strategy: well-being therapy. J Behav Ther Exp Psychiatry $2003 ; 34: 45-63$. 
Fava GA, Ruini C, Linden M: Die Bedeutung des Wohlbefindens in der Behandlung körperlicher und psychischer Erkrankungen; in Linden M, Weig W (eds): Salutotherapie in Prävention und Rehabilitation. Köln, Deutscher Ärzte-Verlag, 2009.

- Forman EM, Herbert JD, Moitra E, Yeomans PD, Geller PA: A randomized controlled effectiveness trial of acceptance and commitment therapy and cognitive therapy for anxiety and depression. Behav Modif 2007;31:772-799.

Freyhan FA: Psychopharmacology and the controversial clinician; in Uhr L, Miller TG (eds): Drugs and Behavior. New York, John Wiley and Sons, 1960, pp 184-198.

Fuchs T: Neurobiology and psychotherapy: an emerging dialogue. Curr Opin Psychiatry 2004;17:479-485.

Glavin GB: Selective noradrenaline depletion markedly alters stress responses in rats. Life Sci 1985; 37:461-465.

Gloaguen V, Cottraux J, Cucherat M, Blackburn I: A meta-analysis of the effects of the cognitive therapy in depressed patients. J Affect Disord 1998;49: 59-72.

Grosscup SJ, Lewinson PM: Unpleasant and pleasant events, and mood. J Clin Psychol 1980;36:252-259.

Goldfried MR, D'Zurilla TJ: A behavioral-analytic model for assessing competence; in Spielberger CD (ed): Current Topics in Clinical and Community Psychology. Academic Press, New York, 1969, pp 151-96.

Hautzinger M: Kognitive Verhaltenstherapie bei Depressionen. Weinheim, Beltz, 2013.

Hayes SC, Strosahl KD, Wilson KG: Acceptance an Commitment Therapy: An Experiential Approach to Behavior Change. New York, Guilford Press, 1999.

Hersen M, Himmelhoch JM, Thase ME, Bellack AS Effects of social skill training, amitiptyline, and psychotherapy in unipolar depressed women. Behav Ther 1984;15:21-40.

Hinsch R, Pfingsten U: Gruppentraining sozialer Kompetenzen. Weinheim, Beltz, 2002.

-Hollon SD, Rubeis RJ, Shelton RC, Amsterdam JD, Salomon RM, O'Reardon JP, Lovett ML, Young, PR, Haman KL, Freeman BB, Gallop R: Prevention of relapse following cognitive therapy vs. medications in moderate to severe depression. Arch Gen Psychiatry 2005;62:417-422.

- Jacobson NS, Dobson KS, Truax PA, Addis ME, Koerner K, Gollan JK, Gortner E, Prince SE: A component analysis of cognitive-behavioral treatment for depression. J Consult Clin Psychol 1996;64: 295-304.

Jacobson NS, Martell CR, Dimidjian S: Behavioral activation treatment for depression: returning to contextual roots. Clin Psychol - Sci Pr 2001;8:255-270.

Kabat-Zinn J: Full Catastrophe Living: Using the Wisdom of Your Body and Mind to Face Stress, Pain and Illness. New York, Delacorte, 1990.

Kanter JW, Manos RC, Busch AM, Rusch LC: Making behavioral activation more behavioral. Behav Modif 2008:32:6:780-803.

Keller MB, McCullough JP, Klein DN, Arnow B, Dunner DL, Gelenberg AJ, Markowitz JC, Nemeroff CB, Russell JM, Thase ME, Trivedi MH, Zajecka JA: Comparison of nefazodone, the cognitive behavioral analysis system of psychotherapie, and their latest combination for the treatment of chronic depression. N Engl J Med 2000;342:1462-1470.

Kiesler DJ: Contemporary Interpersonal Theory and Research: Personality, Psychopathology, and Psychotherapy. New York, John Wiley and Sons, 1996.
Klein DN, Santiago NJ, Vivian D, Blalock JA, Kocsis JH, Markowitz JC, McCullough Jr JP, Rush AJ, Trivedi MH, Arnow BA, Dunner DL, Manber R, Rothbaum B, Thase ME, Keitner GI, Miller IW, Keller MB: Cognitive behavioral analysis system of psychotherapy as a maintenance treatment for chronic depression. J Consult Clin Psychol 2004; 71:681-688.

Klerman GL, Weissman MM, Rounsaville BJ, Chevron ES: Interpersonal Psychotherapy of Depression. New York, Basic Books, 1984.

Kocsis JH, Rush AJ, Markowitz JC, Borian FE, Dunner DL, Koran LM, Klein DN, Trivedi MH, Arnow B, Keitner G, Kornstein SG, Keller MB: Continuation treatment of chronic depression: a comparison of nefazodone, cognitive behavioral analysis system of psychotherapy, and their combination. Psychopharmacol Bull 2003;37:73-87.

Kohlenberg RJ, Kanter JW, Bolling MY, Parker CR, Tsai M: Enhancing cognitive therapy for depression with functional analytic psychotherapy: treatment guidelines and empirical findings. Cogn Behav Pract 2002;9:213-229.

Kohlenberg RJ, Tsai M: Functional Analytic Psychotherapy. Creating Intense and Curative Therapeutic Relationships. New York, Springer, 2007.

Kohlenberg RJ, Tsai M, Parker CR, Bolling MY, Kanter JW: Focusing on the client-therapist interaction. Functional analytic psychotherapy: a behavioral approach. European Psychotherapy 1999;1: $15-24$.

Kuyken W, Byford S, Byng R, Dalgleish T, Lewis G, Taylor R, Watkins ER, Hayes R, Lanham P, Kessler D, Morant N, Evans A: Study protocol for a randomized controlled trial comparing mindfulness-based cognitive therapy with maintenance anti-depressant treatment in the prevention of depressive relapse/recurrence: the PREVENT trial. Trials 2010;11:99.

Kuyken W, Taylor RS, Evans A, Byford S, Watkins E, Holden E, White K, Byng R, Mullan E: Mindfulness-based cognitive therapy to prevent relapse in recurrent depression. J Consult Clin Psychol 2008; 76:966-978.

Lau MA, Segal ZV, Williams JMG: Teasdale's differential activation hypothesis: implication for mechanisms of depressive relapse and suicidal behavior. Behav Res Ther 2004;42:1001-1017.

Lazarus RS, Launier R: Stressbezogene Transaktion zwischen Person und Umwelt; in Nitsch JR (ed): Stress Theorien, Untersuchungen, Massnahmen. Bern, Huber, 1981, pp 213-259.

Lazarus RS, Folkman S: Stress, Appraisal and Coping. New York, Springer, 1984.

Lewinsohn PM: A behavioral approach to depression; in Friedman RJ, Katz MM (eds): The Psychology of Depression: Contemporary Theory and Research. Washington D.C., V.H. Winston and Sons, 1974

Lewinsohn PM, Amenson CS: Some relations between pleasant and unpleasant mood-related events and depression. J Abnorm Psychol 1978;87:644-654.

Lewinsohn PM, Talkington J: Studies on the measurement of unpleasant events and relations with depression. Appl Psych Meas 1979;3:83-101.

Lewinsohn PM, Antonuccio DO, Steinmetz-Breckenridge JL, Teri L: The Coping with Depression Course: A Psychoeducational Intervention for Unipolar Depression. Eugene, Castalia, 1984

Libet J, Lewinsohn PM: The concept of social skill with special reference to the behavior of depressed persons. J Consult Clin Psychol 1973;40:304-312.

Linden DE: How psychotherapy changes the brain-the contribution of functional neuroimaging. Mol Psychiatry 2006;11:6:528-538.
Linden DE: Brain imaging and psychotherapy: methodological considerations and practical implications. Eur Arch Psychiatry Clin Neurosci 2008; 258:71-75.

Linden M: Depression als aktives Verhalten; in Hoffmann N (ed): Depressives Verhalten. Salzburg, Müller, 1976.

Linehan MM: Dialectical behavior therapy for borderline personality disorder: Theory and method. Bull Menninger Clin 1987;51:261-276.

Luty SE, Carter JD, McKenzie JM, Rae AM, Frampton CM, Mulder RT, Joyce PR: Randomised controlled trial of interpersonal psychotherapy and cognitive-behavioural therapy for depression. Br J Psychiatry 2007;190:496-502.

Lyubomirsky S, Tkach C: The consequences of dysphoric rumination; in Papageorgiou C, Wells A (eds): Depressive Rumination: Nature, Theory, and Treatment of Negative Thinking in Depression. Chichester, John Wiley and Sons, 2003, pp 21-41.

Macaskill ND, Macaskill A: Rational-emotive therapy plus pharmacotherapy vs pharmacotherapy alone in treatment of high cognitive dysfunction depression. Cognitive Ther Res 1996;20:80-84.

Mahoney MI: Cognition and Behavior Modification. Cambridge, Ballinger, 1974, p 212.

Markowitz JC, Kocsis JH, Bleiberg KL, Christos PJ, Sacks M: A comparative trial of psychotherapy and pharmacotherapy for 'pure' dysthymic patients. J Affect Disord 2005;89:167-175.

Martell CR, Addis ME, Jacobson NS: Depression in Context: Strategies for Guided Action. New York, Norton, 2001.

Mazzucchelli T, Kane R, Rees C: Behavioral activation treatments for depression in adult: a metaanalysis. Clin Psychol 2009;16:389-411.

McCullough JP: Psychotherapie der chronischen Depression. Cognitive Behavioral Analysis System of Psychotherapy - CBASP. München, Elsevier, 2006.

Michalak J, Heidenreich T, Meibert P, Schulte D: Mindfulness predicts relapse/recurrence in major depressive disorder after mindfulness based cognitive therapy. J Nerv Ment Dis 2008;196:630-633.

Mynors-Wallis LM, Gath DH, Day A, Baker F: Randomised controlled trial for problem solving treatment, antidepressant medication, and combined treatment for major depression in primary care. BMJ 2000;320:26-30.

Mynors-Wallis LM, Gath DH, Lloyd-Thomas AR, Tomlison D: Randomised controlled trial comparing problem solving treatment with amitriptyline and placebo for major depression in primary care. BMJ 1995;310:441-445.

Nemeroff CB, Heim CM, Thase ME, Klein DN, Rush AJ, Schatzberg AF, Ninan PT, McCullough, JP Jr, Weiss PM, Dunner DL, Rothbaum BO, Kornstein S, Keitner G, Keller MB: Differential responses to psychotherapy versus pharmacotherapy in patients with chronic forms of major depression and childhood trauma. Proc Natl Acad Sci USA 2003;100: 14293-14296.

Nezu AM, Pierri MG: Social problem-solving therapy for unipolar depression: an initial dismantling investigation. J Consult Clin Psychol 1989;57:408-413.

Nezu AM, Nezu CM, Perri MG: Problem-Solving Therapy for Depression. Theory, Research, and Clinical Guidelines. New York, John Wiley and Sons, 1989.

Nolen-Hoeksema S: Sex differences in unipolar depression. Psychol Bull 1987;101:259-282.

Nolen-Hoeksema S: The role of rumination in depressive disorders and mixed anxiety / depressive symptoms. J Abnorm Psychol 2000;109:504-511. 
Piaget J: Intelligenz und Affektivität in der Entwicklung des Kindes. Frankfurt/M., Suhrkamp, 1995.

Pretzer JL, Beck AT: A cognitive theory of personality disorders; in Clarkin JF, Lenzenweger MF (eds): Major Theories of Personality Disorder. New York, Guilford Press, 1996, pp 36-105.

Raes F, Williams JMG: The relationship between mindfulness and uncontrollability of ruminative thinking. Mindfulness 2010;4:199-203.

Rehm LP: A self-control model of depression. Behav Ther 1977;8:787-804.

Rehm LP, Adams JH: Self-management; in O'Donohue WT, Fisher JF (eds): Cognitive Behavior Therapy. Applying Empirically Supported Techniques in Your Practice. Hoboken, Wiley, 2003, pp 354-360.

Rehm LP, Fuchs CZ, Roth DM, Kornblith SJ, Romano JM: A comparision of self-control and assertion skills treatments for depression. Behav Ther 1979;10:429-442.

Roth D, Bielski R, Jones M, Parker W, Osborn G: A comparison of self-control therapy and combined self-control therapy and antidepressant medication in the treatment of depression. Behav Ther 1982; 13:133-144.

Ruini C, Fava GA: Clinical applications of well-being therapy; in Linley PA, Joseph S (eds): Positive Psychology in Practice. Hoboken, John Wiley and Sons, 2004.

Ruiz FJ: A review of acceptance and commitment therapy (ACT) empirical evidence: correlational experimental psychopathology, component and outcome studies. International Journal of Psychology and Psychological Therapy 2010;10:125-162.

Ryff CD: Happiness is everything, or is it? Explorations on the meaning of psychological well-being. J Pers Soc Psychol 1989;57:1069-1081.

Schramm E: Interpersonelle Psychotherapie. Stuttgart, Schattauer, 1996.

-Schramm E, Schneider D, Zobel I, van Calker D, Dykierek P, Kech S, Härter M, Berger M: Efficacy of interpersonal psychotherapy plus pharmacotherapy in chronically depressed inpatients. J Affect Disord 2008;109:65-73.

Schramm E, Zobel I, Dykierek P, Kech S, Brakemeier E, Külz A, Berger M: Cognitive behavioral analysis system of psychotherapy versus interpersonal psychotherapy for early-onset chronic depression: a randomized pilot study. J Affect Disord 2010;129: 109-116.
Segal Z, Williams JMG, Teasdale JD: MindfulnessBased Cognitive Therapy for Depression: A New Approach to Preventing Relapse. New York, Guilford Press, 2001.

Segal ZV, Williams MG, Teasale JD: Die Achtsamkeitsbasierte Kognitive Therapie der Depression: Ein neuer Ansatz zur Rückfallprävention. Tübingen, dgvt, 2008.

Seligman MEP: Depression and learned helplessness; in Friedman RJ, Katz MM (eds): The Psychology of Depression. Contemporary Theory and Research. Washington D.C., V.H. Winston and Sons, 1974, pp 83-125.

Seligman MP, Maier SF: Failure to escape traumatic shock. J Exp Psychol 1967;74:1-9.

Segrin C: Social skills deficits associated with depression. Clin Psychol Rev 2000;20:379-403.

Skinner BF: On the conditions of elicitation of certain eating reflexes. Proc Natl Acad Sci USA 1930;16: 433-438.

Sperry L, Carlson J: Psychopathology and Psychotherapy. From DSM-IV Diagnosis to Treatment. Philadelphia, Routledge, 1996.

Sullivan HS: The Interpersonal Theory of Psychiatry. New York, WW Norton \& Co, 1953.

Sulz SKD: Supervision, Intervision und Intravision in Praxis, Klinik, Ambulanz und Ausbildung. München, CIP-Medien, 2007.

Teasdale JD: Cognitive vulnerability to persist depression. Cognition and Emotion 1988;2:247-274.

Teasdale JD, Segal ZV, Williams JMG, Ridgeway VA, Soulsby J, Lau MA: Prevention of relapse/recurrence in major depression by mindfulness-based cognitive therapy. J Consult Clin Psychol 2000; 68:615-623.

Uhmann S, Hoyer J: Metakognitive Therapie der Depression. Psychotherapie 2011;16:306-313.

Wang C, Jia F, Fang R: Comparative study of rationalemotive therapy for 95 patients with dysthymic disorder. Chinese Mental Health Journal 1999;13: 172-183.
Weiss JM, Glazer HI: Effects of acute exposure to stressors on subsequent avoidance-escape behavior. Psychosom Med 1975;37:499-521.

Weiss JM, Glazer HI, Pohorecky LA, Brick J, Miller NE: Effects of chronic exposure to stressors on avoidance-escape behavior and on brain norepinephrine. Psychosom Med 1975;37:522-534.

Wells A: Metacognitive Therapy for Anxiety and Depression. New York, Guilford Press, 2009.

Wells A: Metakognitive Therapie bei Angststörungen und Depressionen. Weinheim, Beltz, 2011.

Wells A, Matthews G: Attention and Emotion: A Clinical Perspective. Hove, Lawrence Erlbaum Associates Ltd., 1994.

Wells A, Fisher P, Myers S, Wheatley J, Patel T, Brewin C: Metacognitive therapy in recurrent and persistent depression: a multiple-baseline study of a new treatment. Cognitive Ther Res 2009;33: 291-300.

Wells A, Fisher P, Myers S, Wheatley J, Patel T, Brewin C: Metacognitive therapy in treatment-resistant depression: a platform trial. Behav Res Ther 2012;50:367-373.

Wilkinson M: Coming into Mind - the Mind-Brain Relationship. Routledge, New York, 2006.

Wolpe J: Psychotherapy by Reciprocal Inhibition. Stanford University Press, California, 1958.

World Health Organization (WHO): International Classification of Functioning, Disability and Health (ICF). Genf, WHO, 2001.

Young JE: Cognitive Therapy for Personality Disorder: A Schema-Focused Approach. Sarasota, Professional Resource Exchange Inc., 1990.

Young JE, Klosko JS, Weishaar ME: Schematherapie. Ein praxisorientiertes Handbuch. Paderborn, Junfermann, 2005.

Young JE, Mattila DE: Schema-focused therapy for depression; in Reinecke MA, Davison MR (eds) Comparative Treatments for Depression. New York, Springer, 2002.

Zettle RD, Rains JC, Hayes SC: Processes of change in acceptance and commitment therapy and cognitive therapy for depression: a mediation reanalysis of Zettle and Rains. Behav Modif 2011;35:265-283. 